\title{
LncRNA NEAT1 Regulates Cementogenic Differentiation of Periodontal Ligament Stem Cells Under Hypoxia by Targeting miR-214-5p/SMAD4 in an HIF-1a-Dependent Manner
}

Yu Ye

Nanjing Medical University https://orcid.org/0000-0003-4487-4597

\section{Liguang Hou}

Nanjing Medical University

Hui Tang

Nanjing Medical University

Liu Liu

Nanjing Medical University

Yan Xu

Nanjing Medical University

Jinhua Yu ( $\nabla$ yujinhua@njmu.edu.cn )

Nanjing Medical University and Jiangsu Province Engineering Research Center of Stomatological

Translational Medicine https://orcid.org/0000-0003-4874-9910

\section{Research}

Keywords: hypoxia, periodontal ligament stem cells, cementogenic differentiation long noncoding RNA, miR-214-5p

Posted Date: July 20th, 2021

DOl: https://doi.org/10.21203/rs.3.rs-715687/v1

License: (c) (i) This work is licensed under a Creative Commons Attribution 4.0 International License. Read Full License 


\section{Abstract}

Background: Human periodontal ligament stem cells (PDLSCs)-mediated regenerative periodontal therapy is a promising method for periodontitis. Besides, odontogenic stem cells are exposed to hypoxia in both physiological and pathological conditions. The behaviour of stem cells under hypoxia is worth exploring. So far, whether long noncoding RNAs (IncRNAs) can affect the proliferation and cementogenesis of PDLSCs, as well as their specific mechanism, remains to be elucidated. This study investigates IncRNA NEAT1 and its possible regulatory mechanisms of cementogenic differentiation of PDLSCs under hypoxia.

Methods: CCK-8 assay, flow cytometry (FCM) and EdU assay was adopted to test the proliferation ability. Osteoblastic/cementogenic differentiation of PDLSCs under hypoxia was detected by western blot assay (WB), quantitative real-time polymerase chain reaction (RT-PCR), alkaline phosphatase (ALP) activity, alcian blue staining (ABS), alizarin red staining (ARS). The relationship between them was proved by dual-luciferase reporter assay and rescue experiments.

Results: PDLSCs exhibited a more powerful cementogenic differentiation potential in hypoxic conditions. miR-214-5p may regulate SMAD4, while Lnc NEAT1 acts as a sponge, forming a ceRNA mechanism in which HIF-1a plays an important role. It may act as an enabler, jointly constitute the regulatory axis of Lnc NEAT1/ miR-214-5p/ SMAD4, and affect the cementogenic differentiation of PDLSCs under hypoxia.

Conclusions: Our results showed that hypoxia could enhance the cementogenic differentiation potential of PDLSCs in vitro. In addition, we found that Lnc NEAT1 may be a potential target for improving the function of PDLSCs in regenerative periodontiology, and Lnc NEAT1/miR-214-5p/SMAD4 could regulate the cementogenic differentiation ability of PDLSCs under hypoxia.

\section{Background:}

Periodontitis is an inflammatory disease characterized by the destruction of periodontal tissue. Bacteria and inflammatory microenvironment may promote this process, leading to cementum, periodontal ligament, and alveolar bone destruction. (1) As one of the most prevalent oral diseases, the pathogenesis and treatment of periodontitis have been concerned. The current treatment strategy focus on obtaining periodontal tissue regeneration. (2) Among them, alveolar bone regeneration and cementum regeneration are the most concerned and challenging links. (3) As a kind of multipotent stem cells, periodontal ligament stem cells (PDLSCs) are located around the blood vessels of alveolar bone and periodontal fibers. (4) It can express the typical markers of mesenchymal stem cells (MSCs), such as STRO-1, CD29, CD90 and CD105. (5) It was found that the PDLSCs may be differentiated into cementum-like cells, periodontal ligament (PDL)-like fibroblasts and osteoblast-like cells, which are involved in reconstructing alveolar bone and root cementum in vitro and in vivo studies. (6) PDLSCs are vital for the formation of alveolar bone and cementum from the perspective of periodontal regeneration. However, the limitation and difficulty of cementum reconstruction should be noted. 
The changes of specific proteins could be used as the markers of alveolar bone and cementum regeneration. During osteo/odontogenesis, the expression of alkaline phosphatase (ALP), osterix (OSX), osteocalcin (OCN), run related transcription factor 2 (Runx2) were up-regulated. (7) Cementum protein-1 (CEMP1) and ptpla / cementum attachment protein (CAP) were specifically expressed in the cementogenesis process. (8) Meanwhile, the major organic component of cementum is collagen, including type I collagen (COL1) accounted for $90 \%$, plays an important role. (9)

It is worth mentioning that the osteoblast markers and cementoblast markers were changed by contrary in most studies, suggesting that the osteogenic/cementogenic process may have an antagonistic effect during the differentiation. A study showed that the increased expression of CEMP-1 promoted cementum formation, while the expressions of osteogenic markers were decreased. (8)

The microenvironment of stem cell growth is the key factor to determine the characteristics of stem cells. It is found that the oxygen concentration in organs or tissues is $1 \%-5 \%$. (9) Under pathological conditions, the oxygen tension could be reduced to $1 \%$. (10) The oxygen concentration in the pulp of lower incisors of rats is about $3 \%-12 \%$. (11) In periodontitis, the oxygen content in the bottom of the periodontal pocket decreased with the increase of probing depth. When the probing depth was 5-10 $\mathrm{mm}$, the oxygen concentration in the bottom of the periodontal pocket was as low as $1.8 \%$. (12) Thus, hypoxia is a common phenomenon in vivo. Odontogenic stem cells are in a hypoxic microenvironment, whether in physiological or pathological conditions.

The effect of hypoxia on the osteo/odontogenesis and cementogenesis of stem cells is controversial. Research has shown that hypoxia can induce human bone marrow mesenchymal stem cell proliferation. (13) Hypoxia tension in the process of cell culture may promote bone mineralization and vascularization. $(14,15)$ The osteogenesis ability of bone marrow-derived stem cells (BMSCs) decreased under hypoxia. (16) Some studies have reported that hypoxia has no or negative effect on the proliferation and survival of dental stem cells. $(17,18)$ Some studies suggest that hypoxia may positively affect the osteogenic/odontogenic differentiation of dental stem cells $(19,20)$, while similar studies have reported the opposite conclusions. (21) However, a few studies in vitro have found hypoxia-inducible factor-1 a (HIF-1a) stimulated the expression of CEMP-1 and enhanced mineralization in PDLSCs, while osteogenesis did not occur. (22) Therefore, it is of great significance to study the cementogenic differentiation of PDLSCs for periodontal regeneration.

At present, it has been found that there are many signalling pathways related to osteoblast/cementogenic differentiation of stem cells, including TGF- $\beta /$ BMP, Wnt/ $\beta$-catenin, Notch, Hedgehog, MAPK, FGF pathway and so on. In the previous study, we conducted bioinformatics analysis of PDLSCs in hypoxia or normoxia and applied high-throughput RNA sequencing (RNA-SEQ) to predict the RNA interaction to clarify the key regulatory factors for PDLSCs behaviour under hypoxia. In these interactions, TGF- $\beta$ /BMP signalling has been proved to initiate periodontal tissue development. (23) SMAD4 is a mediator of the typical TGF- $\beta$ signalling pathway and is crucial in root development. It is found that SMAD4 could 
mediate epithelial-mesenchymal transformation (EMT) during root development by regulating a series of target genes, thereby affecting dentin development. (24)

Noncoding RNA (Noncoding RNAs, ncRNAs) is an important part of gene expression and signal transduction, involving a variety of cellular processes and diseases. (25) Previous studies have shown that miRNAs are involved in a variety of biological processes, including cell growth, migration, apoptosis and differentiation, mainly through binding to the 3 '-untranslated region (3'-UTR) of target genes. $(26,27)$ In particular, many published studies have shown that miRNAs play a key role in osteogenic differentiation. (28) Such as, miR-690 regulates the osteogenic differentiation of $\mathrm{C} 2 \mathrm{C} 12$ cells by targeting p65. (29) miR-144-3p regulates C3H10T1/2 cells by targeting SMAD4, (30) while miR-214 plays a key role in inhibiting bone formation by directly targeting the activation of transcription factor 4. (31) High level of miR-214 seems to delay chondrogenesis, and miR-214 affects chondrocyte differentiation by targeting ATF4. (32) It has been reported that miR-214 inhibits osteogenic differentiation of human PDLSCs by targeting ATF4. (33) Recent studies have shown that IncRNAs could mediate cell differentiation, cell survival, pro-inflammatory and anti-inflammatory processes. (34) Several studies have indicated that some IncRNAs may regulate osteogenetic differentiation through interaction with miRNAs or in specific ways. (35) Lnc NEAT1 is generally considered a tumour-related IncRNA (36), but there is also evidence that Lnc NEAT1 is associated with osteogenic differentiation. (37) Studies have shown that NEAT1 promotes osteoblastic differentiation of BMSCs by regulating the miR-29b-3p/BMP1 axis. (38)

This study aims to explore the differentiation fate and characteristics of PDLSCs in the hypoxic microenvironment and explore whether there is a binding effect between Lnc NEAT1/miR-214/SMAD4 in regulating cementogenic differentiation of PDLSCs under the regulation of HIF-1a.

\section{Methods:}

\section{PDLSCs culture}

Human PDLSCs were isolated and cultured to 3-5 passages as previously described. (39) The Ethics Committee of Nanjing Medical University School of Stomatology (NJMU-2018202) approved the study. The primary PDLSCs were isolated by trypsin digestion. The lysed tissue was evenly inoculated into a $6 \mathrm{~cm}$ dish and then cultured in a-minimum primary medium (a-MEM, GIBCO, life technologies, Ma, USA), which $10 \%$ fetal bovine serum (FBS, hyclone, USA), $100 \mu \mathrm{L} / \mathrm{ml}$ streptomycins and $100 \mathrm{U} / \mathrm{ml}$ penicillin were added. After 24 hours, the medium was changed with a fresh medium containing $10 \%$ fetal bovine serum. Take the primary passage cells in the logarithmic growth phase and adjust the cell density to 10$15 \mathrm{cells} / \mathrm{ml}$. Cells were placed in a $37^{\circ} \mathrm{C}, 5 \% \mathrm{CO}_{2}$ incubator. After culturing in a 96-well culture plate for 12 hours, single-cell wells were labelled. After five days, the medium was changed to observe the formation of a single clone, and the cells from a single cell were expanded to more than $10^{7}$. Follow-up experiments collect 3-5 generations of PDLSCs. The cells were cultured in a hypoxic incubator (Thermo Scientific ${ }^{\text {TM }}$ Heracell VIOS 150i, USA), containing $2 \% \mathrm{O}_{2}, 5 \% \mathrm{CO}_{2}, 92 \% \mathrm{~N}_{2}$, at a temperature of $37^{\circ} \mathrm{C}$, and cultured for several days according to experimental design. 


\section{Adipogenic differentiation}

PDLSCs were planted into 12-well plates, and when cell density reached $60-70 \%$, adipogenic induction solution (Cyagen BioSciences Inc.) was added for culture. The plate was cultured in a $5 \% \mathrm{CO}_{2}$ incubator at $37^{\circ} \mathrm{C}$ and replaced with the induction medium every two days. After 28 days of adipogenic induction, $4 \%$ paraformaldehyde (PFA) fixed the cells for 30 minutes and applied Oil Red $O$ to stain the cells at room temperature.

\section{Chondrogenic differentiation}

PDLSCs cultured in a medium dish were collected and placed in a $15 \mathrm{~mL}$ centrifuge tube containing cartilage induction medium (Cyagen Biosciences Inc.) with the tube cap was gently loosened. The cell microspheres were cultured for $3-5$ days in a $37^{\circ} \mathrm{C}, 5 \% \mathrm{CO}_{2}$ incubator, then the cells were transferred into 24-well plates and cultured for 21 days. Chondroblast induction solution was changed every three days.

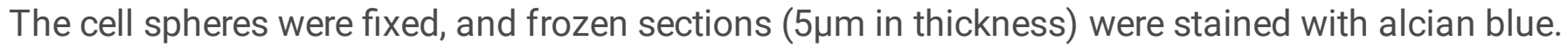

\section{Plasmid construction and cell transfection}

PDLSCs were transfected with miR-214-5p mimics (mimics, $50 \mathrm{nM}$ ), mimics negative control (NC, $50 \mathrm{nM}$ ), miR-214-5p inhibitor (inhibitor, $100 \mathrm{nM}$ ) and inhibitor negative control (iNC, $100 \mathrm{nM}$ ) respectively, with a fusion degree of $30 \%-50 \%$. The transfection reagent is made with the Ribofect ${ }^{\text {TM }} \mathrm{CP}$ Kit (RiboBio, Guangzhou, China). Similarly, PDLSCs transfected by NEAT1, SMAD4, HIF-1a knockout and negative controls (siNEAT1, siSMAD4, siHIF-1a, siNC, $100 \mathrm{nM}$ ) were transfected with the same reagents. By predicting binding sites, the luciferase reporter plasmids of Lnc NEAT1 and SMAD4 and miR-214-5p and Lnc NEAT1 were constructed. 293 T cells were transfected with Lipofectamine 2000 in a serum-free medium for 4-6 hours, and then the basal medium was swapped.

\section{Cell proliferation assay}

PDLSCs were resuspended and inoculated on 96 -well plates $\left(2 \times 10^{3}\right.$ cells/well) in hypoxia or normoxia, respectively. Or transfection of miR-214-5p mimics (mimics, $50 \mathrm{nM}$ ) and mimics negative control (NC, 50 $\mathrm{nM}$ ), miR-214-5p inhibitor (inhibitor, $100 \mathrm{nM}$ ) and inhibitor negative control (iNC, $100 \mathrm{nM}$ ). The same amount of $10 \%$ cell counting kit (CCK)-8 reagents were added to each well on $0,1,3,5$ and 7 days, and the cells were incubated for 2 hours under the same conditions. The absorbance was measured at $450 \mathrm{~nm}$ with a microplate analyzer. Cell-Light ${ }^{\text {TM }}$ EDU Apollo®567 In Vitro Imaging Kit (RiboBio) was adopted to detect EDU. The transfected PDLSCs were inoculated in 24-well plates. After incubated with reagent A for 5 hours, the cells were fixed and treated with one $\times$ Apollo ${ }^{\circledR}$ reaction solution for 30 minutes. The cells were perforated with $0.5 \%$ Triton X-100. The stained nuclei were then incubated with Hoechst 33342 for 30 minutes. After washing with PBS, the staining results were observed with a fluorescence microscope. The cell proliferation rate was calculated by ImageJ software.

\section{Flow cytometry}


When the transfected cells reached $80 \%$, the cells were collected by EDTA-free trypsin (Beyotime, Haimen, China). Flow cytometry was applied to analyze the cell cycle phases (G0/G1, S and G2/M). After being gently washed twice in $0.01 \%$ phosphate-buffered saline (PBS) overnight, the cells were fixed with $75 \%$ precooled ethanol at $4^{\circ} \mathrm{C}$. The proliferation index $\mathrm{PI}=\mathrm{G} 2 \mathrm{M}+\mathrm{S}$ was used to analyze the results. PDLSCs were washed twice with $0.01 \mathrm{~mol} / \mathrm{L}$ PBS and resuspend in $100 \mu \mathrm{L}$ wash buffer to identify the cellular markers of PDLSCs phenotype. PDLSCs were incubated with primary antibodies (CD34, CD45, CD73, CD29, CD90, CD105) for flow cytometry analysis (BD Biosciences, CA, USA).

\section{Quantitative real-time polymerase chain reaction (RT-PCR)}

Total RNA of PDLSCs was extracted with Trizol reagent (Invitrogen, CA, USA). Reverse transcription of RNA was performed by reverse transcriptase. Random primers were used for mRNA and IncRNA, and stem ring primers were used for miRNA. CHAMQ Universal SYBR qPCR Master Mix (Vazyme) was used in ABI 7900 real-time PCR system (Applied Biosystems). Gene expression was normalized by GAPDH (mRNA, IncRNA) or U6 (miRNA) and calculated by $2^{-\triangle} \triangle \mathrm{Ct}$. Each experiment was conducted three times independently. The list of primers is shown in Table 1. 
Table 1

Sense and antisense primers for real-time reverse transcription PCR

\begin{tabular}{|c|c|c|}
\hline Genes & Primers & Sequences $\left(5^{\prime}-3^{\prime}\right)$ \\
\hline \multirow[t]{2}{*}{ GAPDH } & Forward & TCACCAGGGCTGCCATCTGCTCTC \\
\hline & Reverse & TTGCAGTGGCAAAGTGGAGATTGTTG \\
\hline \multirow[t]{2}{*}{ COL1A } & Forward & TCTGACTGGAAGAGCGGAGAG \\
\hline & Reverse & GAGTGGGGAACACACAGGTCT \\
\hline \multirow[t]{2}{*}{ COL3A } & Forward & CTGTGAATCATGCCCTACTGGTC \\
\hline & Reverse & AAGCCTCTGTGTCCTTTCATACC \\
\hline \multirow[t]{2}{*}{ BSP } & Forward & GAACCACTTCCCСАССТTTT \\
\hline & Reverse & ATTCTGACCATCATAGCCATCG \\
\hline \multirow[t]{2}{*}{ NEAT1 } & Forward & GGACAGTAAGCCGAGTGG \\
\hline & Reverse & AGGCAAGCAACACCGAT \\
\hline \multirow[t]{2}{*}{ CAP } & Forward & CCTGGCTCACCTTCTACGAC \\
\hline & Reverse & CCTCAAGCAAGGCAAATGTC \\
\hline \multirow[t]{2}{*}{ CEMP1 } & Forward & GGCGATGCTCAACCTCTAAC \\
\hline & Reverse & GATACCCACCTCTGCCTTGA \\
\hline \multirow[t]{2}{*}{ SMAD4 } & Forward & CTCATGTGATCTATGCCCGTC \\
\hline & Reverse & AGGTGATACAACTCGTTCGTAGT \\
\hline \multirow[t]{2}{*}{ Lnc NEAT1 } & Forward & GGACAGTAAGCCGAGTGG \\
\hline & Reverse & AGGCAAGCAACACCGAT \\
\hline
\end{tabular}

\section{Screening potential pathways involved in hypoxia-mediated cementogenic differentiation}

Based on previous sequencing databases and bioinformatics analysis, enrichment analysis results of PDLSCs differentially expressed target genes in hypoxic microenvironment were screened, and possibly related GO term, and KEGG pathways were screened out.

\section{Western blot analysis}

Protein was extracted, protein concentration was determined, 10\% SDS-PAGE gel was prepared, and protein samples were added. The proteins were separated by $60 \mathrm{~V}$ constant pressure electrophoresis and transferred to the membrane at $300 \mathrm{~mA}$ constant current (Millipore, MA, USA). The membrane was sealed 
with $5 \%$ BSA solution at room temperature for 2 hours. Then the membranes were added anti-HIF-1a (ABCAM, UK), RUNX2 (ABCAM, UK), OCN (ABCAM, UK), GAPDH (Proteintech, USA), COL1A (Proteintech, USA), COL3A (Proteintech, USA), BSP (Santa Cruz, USA), CAP (Santa Cruz, USA), CEMP1 (ABCAM, UK), SMAD3 (Cell Signaling Technology, USA), SMAD4 (Proteintech, USA) and SMAD5 (Proteintech, USA) primary antibodies. The protein bands were detected overnight at $4^{\circ} \mathrm{C}$ and diluted at 1:1000. JV1.8.0 image was used for grey analysis.

\section{Alkaline phosphatase (ALP) activity assay, alizarin red staining (ARS) and alcian blue staining (ABS)}

The alkaline phosphatase assay kit (Jiancheng, Nanjing, China) was used to detect alkaline phosphatase activity. According to the protein concentration of PDLSCs on the seventh day, p-nitrobenzene phosphate was added according to the instructions, and the luminosity value at $405 \mathrm{~nm}$ was measured to calculate the relative ALP activity. When the fusion degree of PDLSCs reached $70 \%$, the cells were inoculated on a 12-well plate and cultured in an osteogenic induction medium containing $0.1 \mathrm{mmol} / \mathrm{L}$ dexamethasone $50 \mu \mathrm{L} / \mathrm{L}$ ascorbic acid and $10 \mathrm{mmol} / \mathrm{L} \beta$-glycerophosphate. After 7 or 14 days of induction, PDLSCs were fixed with $4 \%$ paraformaldehyde for 30 minutes and stained with alkaline phosphatase substrate (Beyotime, Shanghai, China) or alizarin red $(40 \mathrm{mM}, \mathrm{pH}=4.2$, Sigma, Aldrich). The cells were eluted with cetylpyridine chloride $(100 \mathrm{mM})$ for at least 1 hour, and the relative calcium mass was calculated according to the absorbance at $562 \mathrm{~nm}$. PDLSCs were induced by cementogenic differentiation in a 12well plate for 21 days. Cleaning cell surface with PBS, the cells were fixed with methanol for 5 minutes, washed and dried. Alcian blue staining solution A (KeyGEN BioTECH, China) was added and stained for 30 minutes. After washed thoroughly and dried the cells. Alcian blue staining solution $B$ was added for 10 minutes. Cells were washed, dried and observed under a light microscope.

\section{Immunofluorescence staining}

After three days of transfection and induction, PDLSCs were fixed with $4 \%$ paraformaldehyde. Triton X100 (Beijing, China) was treated for 12 minutes and then blocked with goat serum at $37^{\circ} \mathrm{C}$ for 1.5 hours. SMAD4, SMAD5, CEMP1, COL3A or BSP primary antibodies were incubated overnight, and fluorescent secondary antibodies were incubated at room temperature and in the dark for 1.5 hours. DAPI (Beyotime, China) was used to stain the nuclei. Cells were then observed under a fluorescence microscope (Leica, Germany).

\section{Nucleocytoplasmic separation}

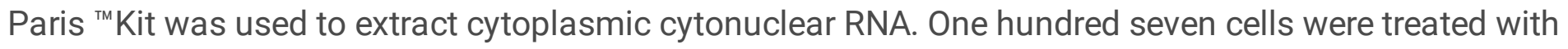
trypsin and centrifuged at low speed. After resuspended and cleaned with PBS, the supernatant was discarded. Resuspend the cells with $400 \mu \mathrm{L}$ Cell Separation Buffer and incubate for 10 minutes. All experiments were carried out on the ice. After centrifugation at $500 \times \mathrm{g}$ for 1 minute, the nuclear precipitation and cytoplasmic supernatant were obtained. Then, after the cytoplasmic supernatant was carefully sucked out, the nuclear residue was dissolved with a $400 \mu \mathrm{L}$ precooled Cell Disruption Buffer. Afterwards, equal amounts of $2 \times$ Lysis/Binding Solution were added to the nuclear and cytoplasmic 
samples at room temperature and immediately and thoroughly mixed by flipping the tubes a few times or gently blowing them. The sample mixture was gently mixed with anhydrous ethanol and centrifuged for 1 minute to pass through the filter. Then, use Wash Solution 1 and $2 / 3$ as instructed to complete the washing step. Finally, the RNA was dissolved in an eluent preheated to $95-100^{\circ} \mathrm{C}$. Nuclear RNA and cytoplasmic RNA were collected for RT-PCR analysis.

\section{Dual-luciferase reporter assay}

HEK-293 T cells were seeded into a 96-well plate $\left(1 \times 10^{5}\right.$ cells/well). When cell density reached $80 \%$, miR214-5p and negative control were transfected into HEK-293 T cells on the next day, respectively. In the meantime, $100 \mathrm{ng}$ Lnc NEAT1 or SMAD4 wild-type reporter plasmid and $20 \mathrm{ng}$ Renilla luciferase (RL) reporter plasmid was co-transfected into HEK-293 T cells. Lnc NEAT1 or SMAD4 reported plasmid mutations were used as controls. A dual-luciferase reporter assay system (Promega, Madison, WI) was used to detect luciferase activity after 48 hours.

\section{Statistical analysis}

Quantitative data were presented as mean \pm standard deviation of three independent records. SPSS17.0 software was used for statistical analysis. T-test or one-way analysis of variance was used to determine that the difference was statistically significant, and $P<0.05$ was considered the standard.

\section{Results:}

\section{Identification of PDLSCs and positive effects of hypoxia on the proliferation and migration of PDLSCs}

1.1 PDLSCs at passage 1 are mostly long fusiform, with an abundant body, uniform cytoplasm, round or oval nucleus located in the center of cells. The cells grew intensively, and colony units were found after monoclonal culture. (Figure S1A) Positive expression of CD90, CD73, CD29, CD105 and negative expression of CD45, CD34 was confirmed in PDLSCs (Figure S1B). Alizarin red, Oil red 0 and alcian blue staining further proved the multidirectional differentiation ability of PDLSCs (Figure S1C). The expression of STRO-1 in PDLSCs was confirmed by fluorescence staining. (Figure S1D)

1.2 To study the effect of hypoxia on the migration ability of PDLSCs, cells were cultured in normoxia and hypoxia conditions. The wound-healing assay results showed that cell migration increased under hypoxia, and wounding healing ability improved $(P<0.001)$. (Figure S2A)

1.3 To investigate hypoxia on the proliferation ability of PDLSCs, CCK8 assay, flow cytometry analysis and EDU assay were carried out. CCK8 assay showed that PDLSCs proliferation increased at three days of hypoxia $(P<0.05)$ and was inhibited five days later $(P<0.001)$. (Figure S2B) Flow cytometry analysis revealed that the proliferation index $(\mathrm{PI}=\mathrm{S} \%+\mathrm{G} 2 \mathrm{M} \%$ ) under hypoxia was higher than normoxia. (Figure S2C) EDU assay confirmed that the number of S-phase fractions of PDLSCs increased under the condition of hypoxia $(P<0.01)$. (Figure S2D) 


\section{Hypoxia inhibited osteogenic differentiation ability of PDLSCs and promoted the expression of cementogenic differentiation markers}

2.1 To detect the effect of hypoxia on the differentiation ability of PDLSCs, cells were cultured under normoxia and hypoxia. $2 \% \mathrm{O}_{2}$ was selected as the experimental condition in the following. The expression of HIF-1a was increased by $2 \% \mathrm{O}_{2}$. (Fig. 1A) The expression of osteogenic markers (Runx2, OCN) was downregulated $(P<0.05)$. (Fig. 1B)

PDLSCs were cultured in the osteogenic inducing fluid and under the condition of normoxia and hypoxia. Alizarin Red Staining was carried out at 14 days. The mineralization of PDLSCs was significantly reduced under hypoxia. (Fig. 1C.a.b) ALP activity was detected seven days later. As an early indicator of PDLSCs' osteogenic differentiation ability, ALP staining of PDLSCs showed that ALP expression lessened and ALP activity decreased significantly under hypoxia $(P<0.01)$. (Fig. 1E)

2.2 To illuminate the effect of hypoxia on the cementogenic differentiation ability of PDLSCs, cells were cultured under normoxia or hypoxia for three days. Results showed that cementogenic differentiation markers (COL1A, COL3A, BSP, CAP and CEMP1) were up-regulated $(P<0.05)$. (Fig. 1D)

Alcian blue staining was used to verify the osteogenic differentiation of PDLSCs. The results showed there were more positive staining in PDLSCs under hypoxia. (Fig. 1C.C)

2.3 To further confirm the differentiation trend of PDLSCs under hypoxia, the changes of cementogenic differentiation indexes were detected at three days. Western blot results showed that the expression of COL1A, COL3A, BSP, CAP and CEMP1 increased three days after hypoxia (Fig. 2A), and immunofluorescence staining results confirmed fluorescent of BSP, COL3A and CEMP1 in PDLSCs increased significantly under hypoxia. (Fig. 2B, C, D)

\section{SMAD4 might be regulated by HIF-1 $a$ in the hypoxia state, thus affecting the cementogenic differentiation of PDLSCs.}

3.1 In the previous study, we sequenced PDLSCs under hypoxia. Based on KEGG pathway analysis and GO term analysis, the relationship between potential pathways and differentially expressed genes was mapped, among which the TGF- $\beta$ pathway is one of the most relevant. (Figure S3)

3.2 The expression changes of SMAD3, SMAD4 and SMAD5 in PDLSCs under hypoxia were detected by Western blot, SMAD 4 was up-regulated, while SMAD3 and SMAD5 were down-regulated. (Fig. 3A) Immunofluorescence results confirmed that the fluorescence expression of SMAD4 increased and SMAD5 decreased in PDLSCs under hypoxia. (Fig. 3B)

3.3 In the hypoxic microenvironment, PDLSCs were treated with SMAD pathway activator kartogenin $(K G N)(1 \mu M)$ and SMAD pathway inhibitor SB525334(1 $\mu \mathrm{M})$. DMSO was used as positive control and the blank group as control. The expressions of COL1A, COL3A, CAP, CEMP1 and SMAD4 increased after KGN stimulation, the expressions of COL1A, COL3A, CAP, CEMP1 and SMAD4 decreased after SB525334 
treatment. (Fig. 3C) To further verify the regulatory effect of SMAD4, the expression of COL1A, COL3A, CAP, CEMP1, and SMAD4 decreased after SMAD4 siRNA treatment. (Fig. 3D) ALP staining and ABS staining results showed that ALP activity increased in the KGN group, and ABS staining positive nodules increased. The number of ABS positive nodules decreased, and ALP activity was inhibited in the SB525334 group. (Fig. 3E, F)

3.4 PDLSCs were treated with one $\mu \mathrm{M}$ of HIF-1a activator Deferoxamine (DFO) under normoxia. The expression levels of COL1A, COL3A, BSP, CAP and CEMP1 in the blank group were increased after DFO stimulation. Meanwhile, the expressions of HIF-1a and SMAD4 were increased. (Fig. 4A) The expression levels of HIF-1a, COL1A, COL3A, CAP, and CEMP1 were decreased after HIF-1a siRNA treatment. (Fig. 4B) To further verify the regulatory effect of SMAD4 under hypoxia, KGN was used as the activator. The expression levels of COL1A, COL3A, BSP, CAP, CEMP1, and SMAD4 were decreased after the cells were treated with siRNA of SMAD4. (Fig. 4C) ALP staining and ABS staining results showed that the activity of ALP was increased in the KGN group, and the number of positive ABS nodules was increased. In the KGN + siSMAD4 group, ALP activity decreased, and ABS staining positive nodules decreased. (Fig. 4C) The expression levels of COL1A, COL3A, BSP, CAP, CEMP1 and SMAD4 were increased after treated with DFO alone or DFO combined with KGN, while the corresponding protein expression levels were decreased after treated with siSMAD4, and the staining results were consistent with the protein results. (Fig. 4D)

\section{Lnc NEAT1/miR-214-5p/SMAD4 may constitute a ceRNA mechanism regulating cementogenic differentiation of PDLSCs}

4.1 PDLSCs were treated with NEAT1 siRNA under hypoxia, the blank group was used as control, and the expressions of COL1A, COL3A, BSP, CAP, CEMP1, and SMAD4 were decreased. RT-PCR was consistent with Western blot results. (Fig. 5A, D) ALP staining and ABS staining results showed that the ALP activity of the siNEAT1 group was decreased, and the number of ABS staining positive nodules decreased. (Fig. 5B) To further verify the regulatory effect of miR-214-5p and SMAD4, a dual-luciferase reporter assay was used to verify the binding sites, and the protein expression of SMAD4 decreased when miR-214-5p was overexpressed. (Fig. 5C) Nucleoplasmic separation experiments verified that Lnc NEAT1 was in the cytoplasm, a prerequisite for the ceRNA mechanism. (Fig. 5E) Dual-luciferase reporter gene assay verified the existence of a binding site between Lnc NEAT1 and miR-214-5p. (Fig. 5F)

4.2 PDLSCs were treated with miR-214-5p mimics under hypoxia, and the NC group was used as control. The protein expression levels of COL1A, COL3A, BSP, CAP, and CEMP1 were decreased. (Fig. 6A) ALP staining and $A B S$ staining results showed that ALP activity was decreased in the mimics group, the number of positive ABS staining nodules were decreased. (Fig. 6B) CCK-8 detection showed that miR-214$5 p$ mimics, inhibitor and corresponding NC had no significant effect on the proliferation of PDLSCs. (Fig. 6C) Cells were treated with miR-214-5p inhibitor under hypoxia, and the iNC group was used as a control. The protein expressions of COL1A, COL3A, BSP, CAP and CEMP1 were increased. (Fig. 6D) ALP staining and $A B S$ staining results showed that the activity of ALP in the inhibitor group was increased, and the number of positive ABS staining nodules were increased. (Fig. 6E) 
4.3 The function was further verified by the rescue experiment. PDLSCs were co-transfected with siNEAT1 and miR-214-5p inhibitor and treated with siNC and miR-214 NC group as control. The results showed that miR-214-5p inhibitor could reverse the expression of COL1A, COL3A, BSP, CAP, CEMP1 after siNEAT1 treatment. (Fig. 7A) The results of RT-PCR and Western blot were consistent. (Fig. 7B) miR-214-5p inhibitor could reverse the decrease of $\angle n c N E A T 1$ gene expression induced by siNEAT1. (Fig. 7C) The results showed that miR-214-5p inhibitor could reverse the expression of COL1A, COL3A, BSP, CAP, CEMP1 in PDLSCs. (Fig. 7D) RT-PCR and Western blot results were consistent. (Fig. 7E) Experimental mechanism diagram: Lnc NEAT1/miR-214-5p/SMAD4 may constitute the mechanism of regulating PDLSCs cementogenesis differentiation. (Fig. 7F)

\section{Discussion:}

The microenvironmental niche of MSCs is a key factor in determining their characteristics, maintaining and regulating their functions. Oxygen in the microenvironment is essential for normal cell growth and differentiation. Under physiological conditions, the oxygen tension of human organs and tissues is between $2 \%$ and $9 \%$. Under pathological conditions or in some areas of relative hypoxia, oxygen tension can be reduced to $1 \%$ or even lower in some cases. $(10,21)$ So far, the concentrations of odontogenic stem cells associated with the hypoxic microenvironment have been reported to be $1 \%, 2 \%, 3 \%, 5 \%$, etc. (40). The average partial pressure of oxygen at the bottom of the periodontal pocket is only $13.3 \mathrm{mmHg}$ $\left(1.8 \% \mathrm{O}_{2}\right)$. $(12)$ In most cases, the oxygen concentration in the periodontal microenvironment is more than $1 \%$, except for extreme pathological conditions. Here, $2 \% \mathrm{O}_{2}$ tension was adopted to study the proliferation and migration of PDLSCs and the cementogenesis potential. It is meant to elucidate the behaviour change and regeneration potential of PDLSCs in hypoxia. The study may help predict the possible molecular mechanism and provide a possible target factor for the regulation of PDLSCs in mediating periodontal tissue regeneration.

Tooth derived stem cells are in a hypoxia environment in vivo. The study found that proliferation, osteogenesis/cementogenic differentiation of hypoxic stem cells could affect tooth development. Hypoxia condition also promotes exocytosis of dental derived stem cells, promoting blood vessels and cell migration. However, a study of hypoxic PDLSCs on cementogenic differentiation is relatively rare. PDLSCs are considered the best cell source in periodontal regeneration, containing self-renewal and multidirectional differentiation potential. PDLSCs can differentiate into cementum, alveolar bone and periodontal membrane tissue, which cementum regeneration is difficult for periodontitis treatment. PDLSCs are widely used in periodontal repair and regeneration treatment of periodontal disease. However, more evidence is needed to clarify what determines and modulates the multilineage differentiation potential of PDLSCs in a hypoxic microenvironment. It has been reported that the expression of CEMP1 is increased under hypoxia (41), and it was found that the HIF-1 activator may have the ability to stimulate root regeneration and bone formation. HIF-1 $\mathrm{a}$ is a marker of the hypoxic microenvironment. (22) This study aims to mimic the hypoxic microenvironment of PDLSCs and explore the regulatory mechanism of cementoblasts regeneration. 
The cementum is a thin layer of mineralized tissue attached to the root's surface, and it is a vital part of periodontal tissue. The identification of cementum and alveolar bone is mainly cementum-specific proteins, such as CAP and CEMP1. In addition, the cementum matrix includes collagen type I and type III, (42) fibronectin, osteopontin (OPN), BSP, OCN, etc. (43)

The cementogenic differentiation of PDLSCs may be in the opposite trend with osteogenic differentiation. Some studies have shown that the osteogenic specific factor of PDLSCs decreased in osteogenic differentiation and increased after vitamin $C$ stimulation. It is found that the formation of cementum-like tissue without bone marrow was found in vivo research of PDLSCs. (44). This study found that the cementogenesis of PDLSCs may be superior to that of osteogenesis under hypoxia.

With the development of transcriptomics, the study of ncRNAs has attracted more and more attention. By controlling the expression level of protein-coding RNA, ncRNAs play an important role in regulating gene expression and are involved in various cellular processes such as cell proliferation, cell differentiation and biological development. (45) Evidence showed that ncRNAs can regulate the differentiation potential of PDLSCs. Thus, it is necessary to study the characteristics, and regulatory mechanism of ncRNAs in hypoxia treated PDLSCs for periodontal regeneration. It is reported that LnCRNA-CCAT1 can directly bind to miR-218, improve the expression of type I collagen, OPN and OCN in dental pulp stem cells (DPSCs), and promote the proliferation and differentiation of DPSCs.(46) CircRNA3140 can synergize with miR-21

and cerebellar degeneration related protein one transcript (CDR1AS) and miR-7 to regulate the osteogenic differentiation of PDLSCs. $(47,48)$ LncRNA-AnCRs competitively bind to miR-758 and inhibit the osteogenic differentiation of PDLSCs (49). In addition, IncRNA-POIR found in periodontitis patients may act as the ceRNA of miR-182 to inhibit the expression of ALP, Runx2 and OSX, thereby inhibiting the osteogenesis of PDLSCs. (50) In our study, we predicted and verified the ceRNA regulatory axis that may regulate the cementogenic differentiation trend of PDLSCs under hypoxia.

This study revealed the increasing cementogenic trend of PDLSCs under the hypoxic microenvironment. Lnc NEAT1/miR-214-5p/SMAD4 may act as a regulatory axis to modulate the cementogenic differentiation of PDLSCs in a HIF-1a dependent manner.

\section{Declarations:}

\section{Ethics approval and consent to participate:}

This study was approved by the Ethics Committee of Nanjing Medical University School of Stomatology (NJMU-2018202).

\section{Consent for publication:}

Not applicable.

\section{Availability of data and materials:}


The datasets generated during the current study are available in the [SRAdatabase/PRJNA684674/https://www.ncbi.nlm.nih.gov/sra/PRJNA684674].

The datasets analyzed during the current study are available from the corresponding author on reasonable request.

\section{Competing interests:}

The authors declare that they have no competing interests.

\section{Funding:}

This work was supported by National Natural Science Foundation of China (grant numbers: 81873707 and 81900962).

\section{Authors' contributions:}

JY and $Y X$ designed the study. YY conducted the experiment. LH, HT, and LL performed and collected the data. YY analyzed the data and wrote the manuscript. All authors read and approved the final manuscript.

\section{Acknowledgements:}

Not applicable.

\section{References:}

1. Hajishengallis G. Periodontitis: from microbial immune subversion to systemic inflammation. Nature reviews Immunology. 2015;15(1):30-44. https://doi.org/10.1038/nri3785.

2. Lee $\mathrm{SI}$, Lee DW, Yun HM, Cha HJ, Bae CH, Cho ES, et al. Expression of thymosin beta-4 in human periodontal ligament cells and mouse periodontal tissue and its role in osteoblastic/cementoblastic differentiation. Differentiation; research in biological diversity. 2015;90(1-3):16-26. https://doi.org/10.1016/j.diff.2015.08.003.

3. Zhang Y, Kong N, Zhang Y, Yang W, Yan F. Size-dependent Effects of Gold Nanoparticles on Osteogenic Differentiation of Human Periodontal Ligament Progenitor Cells. Theranostics. 2017;7(5):1214-24. https://doi.org/10.7150/thno.17252.

4. Seo BM, Miura M, Gronthos S, Bartold PM, Batouli S, Brahim J, et al. Investigation of multipotent postnatal stem cells from human periodontal ligament. Lancet (London, England). 2004;364(9429):149-55. https://doi.org/10.1016/s0140-6736(04)16627-0.

5. Yu Z, Gauthier P, Tran QT, El-Ayachi I, Bhatti FU, Bahabri R, et al. Differential Properties of Human ALP (+) Periodontal Ligament Stem Cells vs Their ALP (-) Counterparts. Journal of stem cell research \& therapy. 2015;5(7). https://doi.org/10.4172/2157-7633.1000292. 
6. Seo BM, Miura M, Sonoyama W, Coppe C, Stanyon R, Shi S. Recovery of stem cells from cryopreserved periodontal ligament. Journal of dental research. 2005;84(10):907-12. https://doi.org/10.1177/154405910508401007.

7. Yamazaki H, Tsuneto M, Yoshino M, Yamamura K, Hayashi S. Potential of dental mesenchymal cells in developing teeth. Stem cells (Dayton, Ohio). 2007;25(1):78-87. https://doi.org/10.1634/stemcells.2006-0360.

8. Komaki M, Iwasaki K, Arzate H, Narayanan AS, Izumi Y, Morita I. Cementum protein 1 (CEMP1) induces a cementoblastic phenotype and reduces osteoblastic differentiation in periodontal ligament cells. Journal of cellular physiology. 2012;227(2):649-57. https://doi.org/10.1002/jcp.22770.

9. Glimcher MJ. Mechanism of calcification: role of collagen fibrils and collagen-phosphoprotein complexes in vitro and in vivo. The Anatomical record. 1989;224(2):139-53. https://doi.org/10.1002/ar.1092240205.

10. Mohyeldin A, Garzón-Muvdi T, Quiñones-Hinojosa A. Oxygen in stem cell biology: a critical component of the stem cell niche. Cell Stem Cell. 2010;7(2):150-61. https://doi.org/10.1016/j.stem.2010.07.007.

11. Yu CY, Boyd NM, Cringle SJ, Alder VA, Yu DY. Oxygen distribution and consumption in rat lower incisor pulp. Archives of oral biology. 2002;47(7):529-36. https://doi.org/10.1016/s00039969(02)00036-5.

12. Mettraux GR, Gusberti FA, Graf H. Oxygen tension ( $\mathrm{pO} 2$ ) in untreated human periodontal pockets. Journal of periodontology. 1984;55(9):516-21. https://doi.org/10.1902/jop.1984.55.9.516.

13. Sakdee JB, White RR, Pagonis TC, Hauschka PV. Hypoxia-amplified proliferation of human dental pulp cells. Journal of endodontics. 2009;35(6):818-23. https://doi.org/10.1016/j.joen.2009.03.001.

14. Li L, Zhu YQ, Jiang L, Peng W, Ritchie HH. Hypoxia promotes mineralization of human dental pulp cells. Journal of endodontics. 2011;37(6):799-802. https://doi.org/10.1016/j.joen.2011.02.028.

15. Aranha AM, Zhang Z, Neiva KG, Costa CA, Hebling J, Nör JE. Hypoxia enhances the angiogenic potential of human dental pulp cells. Journal of endodontics. 2010;36(10):1633-7. https://doi.org/10.1016/j.joen.2010.05.013.

16. Wang Y, Li J, Wang Y, Lei L, Jiang C, An S, et al. Effects of hypoxia on osteogenic differentiation of rat bone marrow mesenchymal stem cells. Molecular and cellular biochemistry. 2012;362(1-2):25-33. https://doi.org/10.1007/s11010-011-1124-7.

17. Zhou Y, Fan W, Xiao Y. The effect of hypoxia on the stemness and differentiation capacity of PDLC and DPC. BioMed research international. 2014;2014:890675. https://doi.org/10.1155/2014/890675.

18. Vanacker J, Viswanath A, De Berdt P, Everard A, Cani PD, Bouzin C, et al. Hypoxia modulates the differentiation potential of stem cells of the apical papilla. Journal of endodontics. 2014;40(9):14108. https://doi.org/10.1016/j.joen.2014.04.008.

19. Ito K, Matsuoka K, Matsuzaka K, Morinaga K, Inoue T. Hypoxic condition promotes differentiation and mineralization of dental pulp cells in vivo. International endodontic journal. 2015;48(2):115-23. https://doi.org/10.1111/iej.12288. 
20. Wu Y, Yang Y, Yang P, Gu Y, Zhao Z, Tan L, et al. The osteogenic differentiation of PDLSCs is mediated through MEK/ERK and p38 MAPK signalling under hypoxia. Archives of oral biology. 2013;58(10):1357-68. https://doi.org/10.1016/j.archoralbio.2013.03.011.

21. Shi R, Yang H, Lin X, Cao Y, Zhang C, Fan Z, et al. Analysis of the characteristics and expression profiles of coding and noncoding RNAs of human dental pulp stem cells in hypoxic conditions. Stem cell research \& therapy. 2019;10(1):89. https://doi.org/10.1186/s13287-019-1192-2.

22. Choi H, Jin H, Kim JY, Lim KT, Choung HW, Park JY, et al. Hypoxia promotes CEMP1 expression and induces cementoblastic differentiation of human dental stem cells in an HIF-1-dependent manner. Tissue engineering Part A. 2014;20(1-2):410-23. https://doi.org/10.1089/ten.TEA.2013.0132.

23. Koba T, Watanabe K, Goda S, Kitagawa M, Mutoh N, Hamada N, et al. The Effect of Transforming Growth Factor Beta 1 on the Mineralization of Human Cementoblasts. Journal of endodontics. 2021;47(4):606-11. https://doi.org/10.1016/j.joen.2020.12.019.

24. Huang X, Xu X, Bringas P, Jr., Hung YP, Chai Y. Smad4-Shh-Nfic signaling cascade-mediated epithelial-mesenchymal interaction is crucial in regulating tooth root development. Journal of bone and mineral research: the official journal of the American Society for Bone and Mineral Research. 2010;25(5):1167-78. https://doi.org/10.1359/jbmr.091103.

25. Zhang J, Hao X, Yin M, Xu T, Guo F. Long non-coding RNA in osteogenesis: A new world to be explored. Bone \& joint research. 2019;8(2):73-80. https://doi.org/10.1302/2046-3758.82.Bjr-20180074.R1.

26. Zhen L, Yun-Hui L, Hong-Yu D, Jun M, Yi-Long Y. Long noncoding RNA NEAT1 promotes glioma pathogenesis by regulating miR-449b-5p/c-Met axis. Tumour biology: the journal of the International Society for Oncodevelopmental Biology and Medicine. 2016;37(1):673-83.

https://doi.org/10.1007/s13277-015-3843-y.

27. Wang P, Liu YH, Yao YL, Li Z, Li ZQ, Ma J, et al. Long non-coding RNA CASC2 suppresses malignancy in human gliomas by miR-21. Cellular signalling. 2015;27(2):275-82. https://doi.org/10.1016/j.cellsig.2014.11.011.

28. Huang C, Geng J, Jiang S. MicroRNAs in regulation of osteogenic differentiation of mesenchymal stem cells. Cell and tissue research. 2017;368(2):229-38. https://doi.org/10.1007/s00441-016-24622.

29. Yu S, Geng Q, Pan Q, Liu Z, Ding S, Xiang Q, et al. MiR-690, a Runx2-targeted miRNA, regulates osteogenic differentiation of C2C12 myogenic progenitor cells by targeting NF-kappaB p65. Cell \& bioscience. 2016;6:10. https://doi.org/10.1186/s13578-016-0073-y.

30. Huang C, Geng J, Wei X, Zhang R, Jiang S. MiR-144-3p regulates osteogenic differentiation and proliferation of murine mesenchymal stem cells by specifically targeting Smad4. FEBS letters. 2016;590(6):795-807. https://doi.org/10.1002/1873-3468.12112.

31. Wang X, Guo B, Li Q, Peng J, Yang Z, Wang A, et al. miR-214 targets ATF4 to inhibit bone formation. Nature medicine. 2013;19(1):93-100. https://doi.org/10.1038/nm.3026. 
32. Roberto VP, Gavaia P, Nunes MJ, Rodrigues E, Cancela ML, Tiago DM. Evidences for a New Role of miR-214 in Chondrogenesis. Scientific reports. 2018;8(1):3704. https://doi.org/10.1038/s41598-01821735-w.

33. Yao S, Zhao W, Ou Q, Liang L, Lin X, Wang Y. MicroRNA-214 Suppresses Osteogenic Differentiation of Human Periodontal Ligament Stem Cells by Targeting ATF4. Stem cells international. 2017;2017:3028647. https://doi.org/10.1155/2017/3028647.

34. Chen YG, Satpathy AT, Chang HY. Gene regulation in the immune system by long noncoding RNAs. Nature immunology. 2017;18(9):962-72. https://doi.org/10.1038/ni.3771.

35. He S, Yang S, Zhang Y, Li X, Gao D, Zhong Y, et al. LncRNA ODIR1 inhibits osteogenic differentiation of hUC-MSCs through the FBX025/H2BK120ub/H3K4me3/OSX axis. Cell death \& disease. 2019;10(12):947. https://doi.org/10.1038/s41419-019-2148-2.

36. Arshi A, Sharifi FS, Khorramian Ghahfarokhi M, Faghih Z, Doosti A, Ostovari S, et al. Expression Analysis of MALAT1, GAS5, SRA, and NEAT1 IncRNAs in Breast Cancer Tissues from Young Women and Women over 45 Years of Age. Molecular therapy Nucleic acids. 2018;12:751-7. https://doi.org/10.1016/j.omtn.2018.07.014.

37. Li H, Xie S, Li H, Zhang R, Zhang H. LncRNA MALAT1 mediates proliferation of LPS treated-articular chondrocytes by targeting the miR-146a-PI3K/Akt/mTOR axis. Life sciences. 2020;254:116801. https://doi.org/10.1016/j.Ifs.2019.116801.

38. Zhang Y, Chen B, Li D, Zhou X, Chen Z. LncRNA NEAT1/miR-29b-3p/BMP1 axis promotes osteogenic differentiation in human bone marrow-derived mesenchymal stem cells. Pathology, research and practice. 2019;215(3):525-31. https://doi.org/10.1016/j.prp.2018.12.034.

39. Xu Y, Wang Y, Pang X, Li Z, Wu J, Zhou Z, et al. Potassium dihydrogen phosphate promotes the proliferation and differentiation of human periodontal ligament stem cells via nuclear factor kappa $B$ pathway. Experimental cell research. 2019;384(1):111593. https://doi.org/10.1016/j.yexcr.2019.111593.

40. Yang A, Wang L, Jiang K, Lei L, Li H. Nuclear receptor coactivator 4-mediated ferritinophagy drives proliferation of dental pulp stem cells in hypoxia. Biochemical and biophysical research communications. 2021;554:123-30. https://doi.org/10.1016/j.bbrc.2021.03.075.

41. Li S, Shao J, Zhou Y, Friis T, Yao J, Shi B, et al. The impact of Wnt signalling and hypoxia on osteogenic and cementogenic differentiation in human periodontal ligament cells. Mol Med Rep. 2016;14(6):4975-82. https://doi.org/10.3892/mmr.2016.5909.

42. Arzate H, Jiménez-García LF, Alvarez-Pérez MA, Landa A, Bar-Kana I, Pitaru S. Immunolocalization of a human cementoblastoma-conditioned medium-derived protein. Journal of dental research. 2002;81(8):541-6. https://doi.org/10.1177/154405910208100808.

43. Zeichner-David M. Regeneration of periodontal tissues: cementogenesis revisited. Periodontology 2000. 2006;41:196-217. https://doi.org/10.1111/j.1600-0757.2006.00162.x.

44. Gauthier P, Yu Z, Tran QT, Bhatti FU, Zhu X, Huang GT. Cementogenic genes in human periodontal ligament stem cells are downregulated in response to osteogenic stimulation while upregulated by 
vitamin C treatment. Cell and tissue research. 2017;368(1):79-92. https://doi.org/10.1007/s00441016-2513-8.

45. Givel AM, Kieffer Y, Scholer-Dahirel A, Sirven P, Cardon M, Pelon F, et al. miR200-regulated CXCL12 $\beta$ promotes fibroblast heterogeneity and immunosuppression in ovarian cancers. Nature communications. 2018;9(1):1056. https://doi.org/10.1038/s41467-018-03348-z.

46. Zhong YX, Li WS, Liao LS, Liang L. LnCRNA CCAT1 promotes cell proliferation and differentiation via negative modulation of miRNA-218 in human DPSCs. European review for medical and pharmacological sciences. 2019;23(9):3575-83. https://doi.org/10.26355/eurrev_201905_17779.

47. Wang H, Feng C, Jin Y, Tan W, Wei F. Identification and characterization of circular RNAs involved in mechanical force-induced periodontal ligament stem cells. Journal of cellular physiology. 2019;234(7):10166-77. https://doi.org/10.1002/jcp.27686.

48. Li X, Zheng Y, Zheng Y, Huang Y, Zhang Y, Jia L, et al. Circular RNA CDR1as regulates osteoblastic differentiation of periodontal ligament stem cells via the miR-7/GDF5/SMAD and p38 MAPK signaling pathway. Stem cell research \& therapy. 2018;9(1):232. https://doi.org/10.1186/s13287018-0976-0.

49. Peng W, Deng W, Zhang J, Pei G, Rong Q, Zhu S. Long noncoding RNA ANCR suppresses bone formation of periodontal ligament stem cells via sponging miRNA-758. Biochemical and biophysical research communications. 2018;503(2):815-21. https://doi.org/10.1016/j.bbrc.2018.06.081.

50. Wang L, Wu F, Song Y, Li X, Wu Q, Duan Y, et al. Long noncoding RNA related to periodontitis interacts with miR-182 to upregulate osteogenic differentiation in periodontal mesenchymal stem cells of periodontitis patients. Cell death \& disease. 2016;7(8): e2327. https://doi.org/10.1038/cddis.2016.125.

\section{Figures}



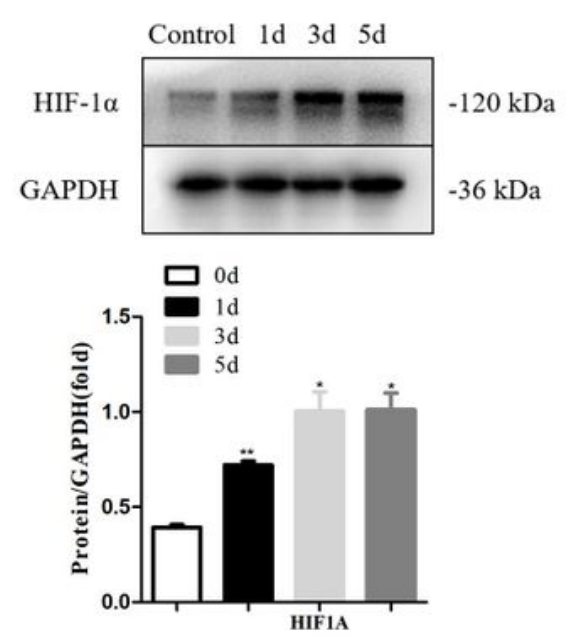

B

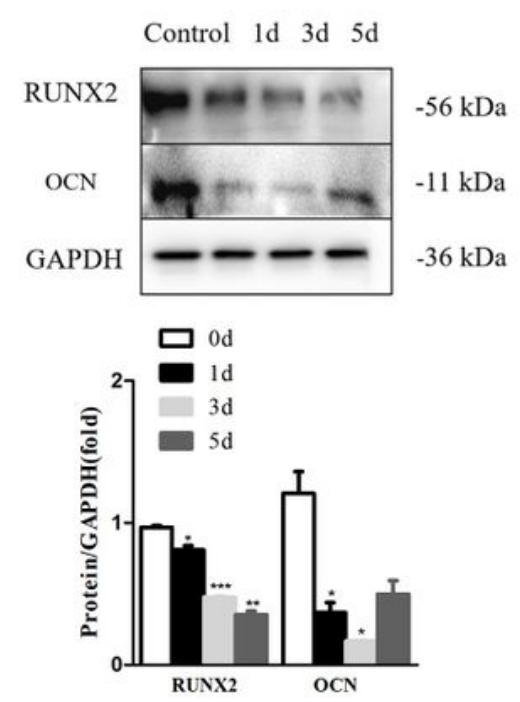

C

(a)

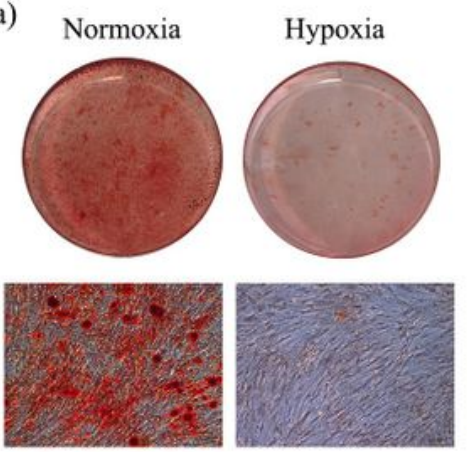

ARS staining (b)

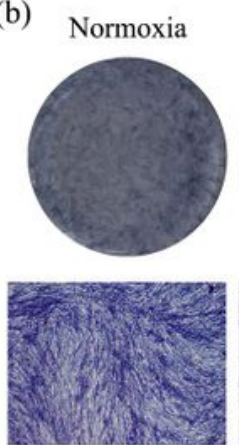

ALP staining
D
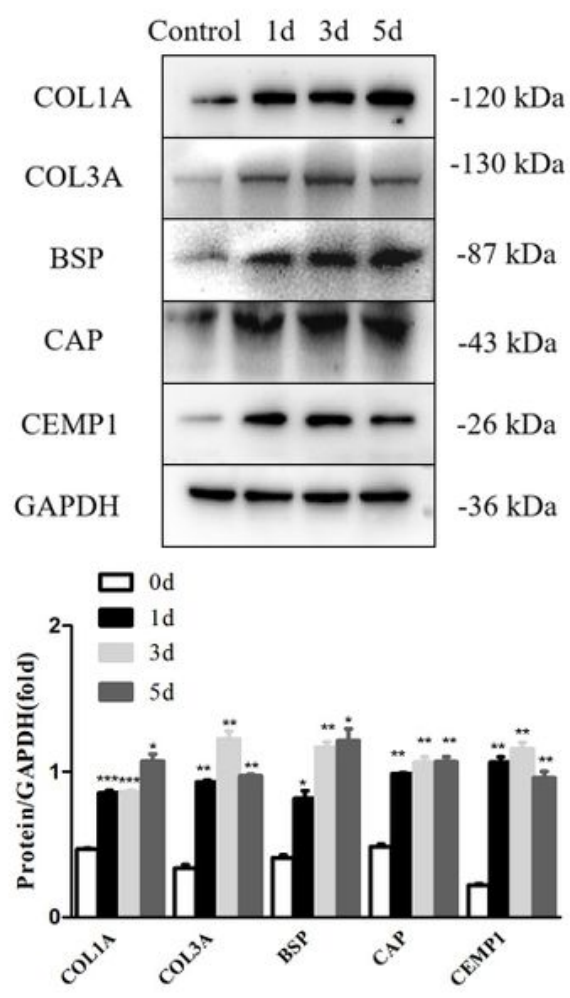

E

ALP activity

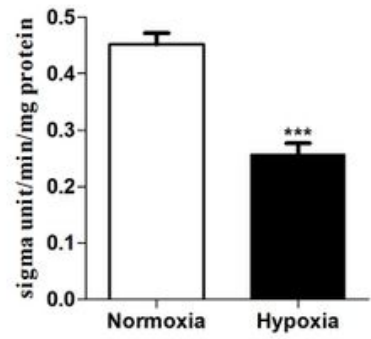

(c) Normoxia Hypoxia
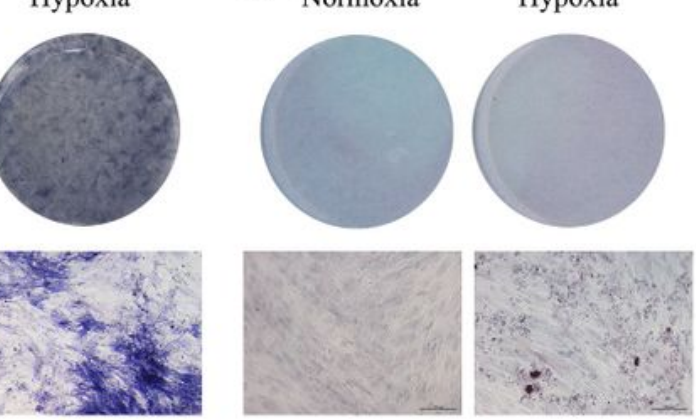

ABS staining

\section{Figure 1}

Hypoxia inhibited osteogenic differentiation ability of PDLSCs and promoted the expression of cementogenic differentiation markers. (A) Western blot analysis detected the expression levels of hypoxia marker protein HIF-1a. GAPDH was used as a reference protein. (B) Western blot showed that RUNX2 and OCN expressions were inhibited by hypoxia after osteogenic induction for 14 days. GAPDH was used as an internal control. (C) (a): Alizarin red staining showed the mineralization of PDLSCs was decreased 
significantly in the hypoxia group after osteogenic induction for 14 days. (b) (E): Hypoxia reduced the ALP activity of PDLSCs 7 days after osteogenic induction. (c): Alcian blue staining showed the cementogenic differentiation of PDLSCs was increased significantly in hypoxia. (D) Western blot showed that cementogenic differentiation marker (COL1A, COL3A, BSP, CAP and CEMP1) were up-regulated. Data are presented as mean $\pm S D ;{ }^{*} P<0.05,{ }^{*} \mathrm{P}<0.01$ and $* * * P<0.001$.

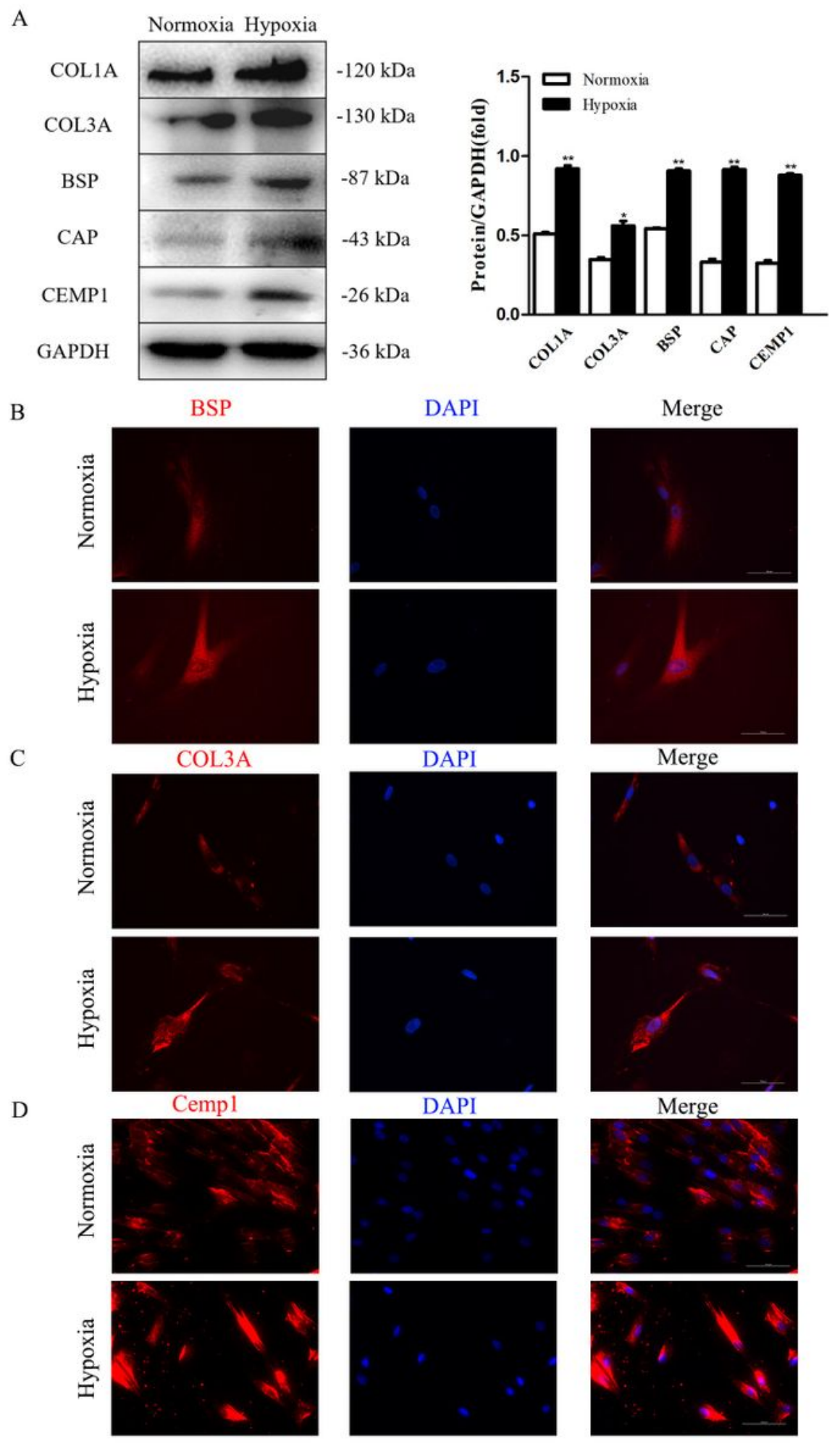

Figure 2 
Hypoxia promoted the expression of cementogenic differentiation markers in PDLSCs. (A) Western blot showed that cementogenic differentiation marker (COL1A, COL3A, BSP, CAP and CEMP1) were upregulated on day 3. (B)(C)(D) Immunofluorescence staining results confirmed that the fluorescent expression of BSP, COL3A and CEMP1 in PDLSCs increased significantly under hypoxia. Data are presented as mean $\pm S D ; * P<0.05$, $* * P<0.01$ and $* * * P<0.001$.

A
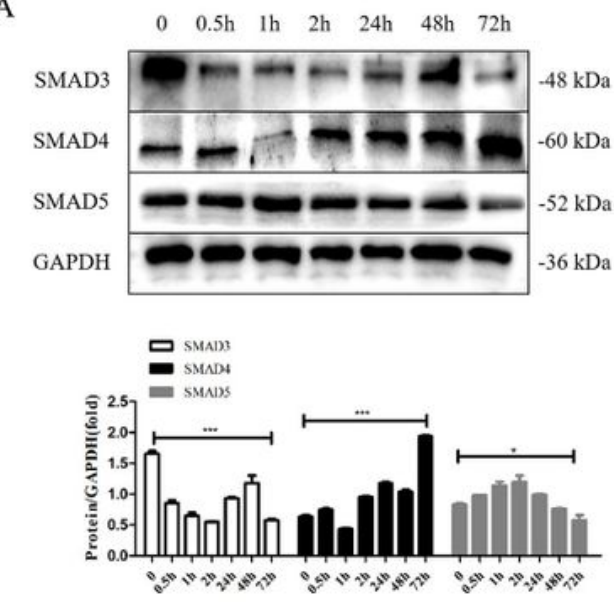

B
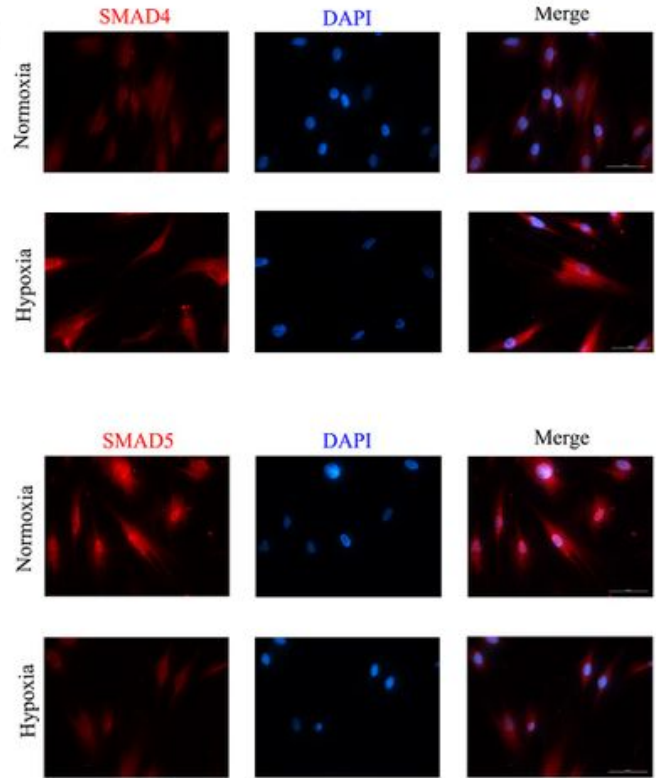

C
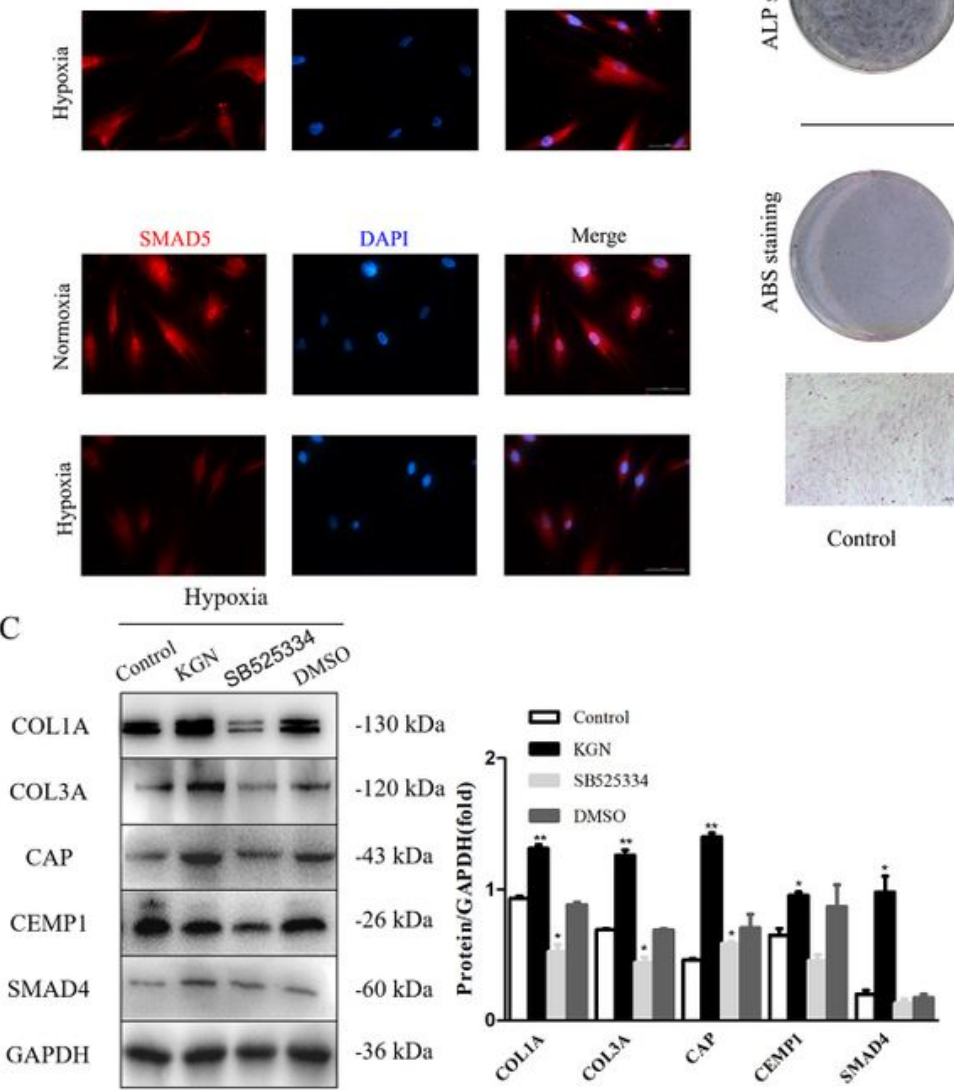

KGN: Kartogenin
D

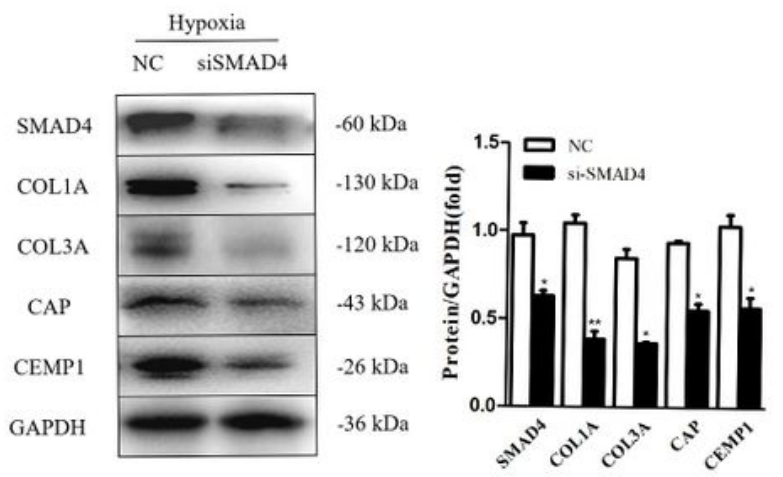

E
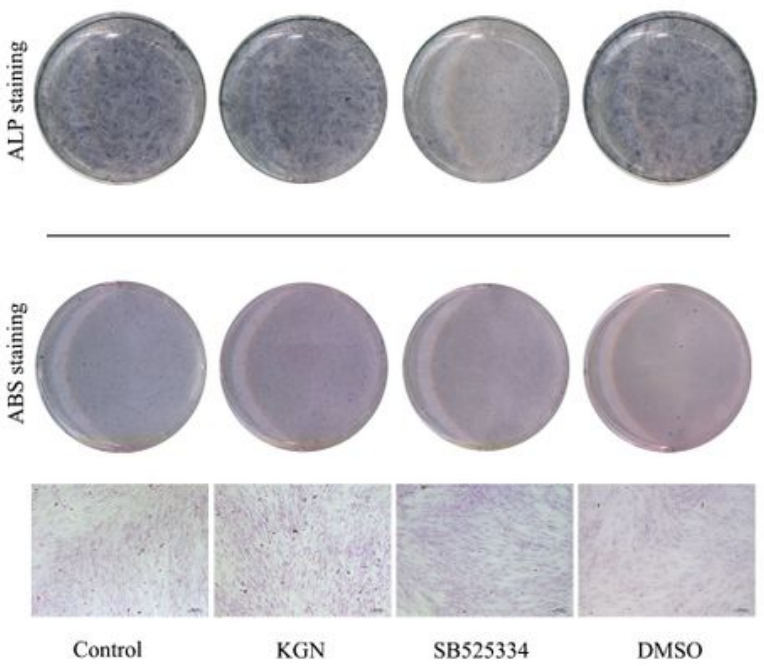

$\mathrm{F}$

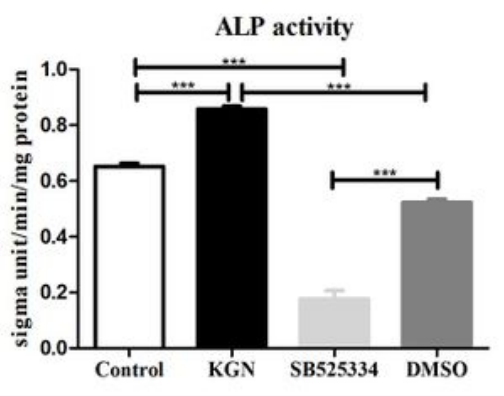

Figure 3 
SMAD4 might be regulated by HIF-1 $a$, thus affecting the cementogenic differentiation of PDLSCs. (A) Western blot detected the expression changes of SMAD3, SMAD4 and SMAD5 in PDLSCs under hypoxia. (B) Immunofluorescence staining showed that the fluorescence expression of SMAD4 increased and SMAD5 decreased in PDLSCs under hypoxia. (C) The expressions of COL1A, COL3A, CAP, CEMP1 and SMAD4 increased after kartogenin (KGN) stimulation, the expressions of COL1A, COL3A, CAP, CEMP1 and SMAD4 decreased after SB525334 treatment. (D) Western blot showed that SMAD4 siRNA treatment decreased the expression of COL1A, COL3A, CAP, CEMP1. (E) (F) ALP staining and ABS staining results showed that ALP activity increased in KGN group and ABS staining positive nodules increased, the trend was opposite in SB525334 group. Data are presented as mean \pm SD; ${ }^{*} P<0.05,{ }^{*} \mathrm{P}<0.01$ and ${ }^{\star \star *} \mathrm{P}<$ 0.001 . 


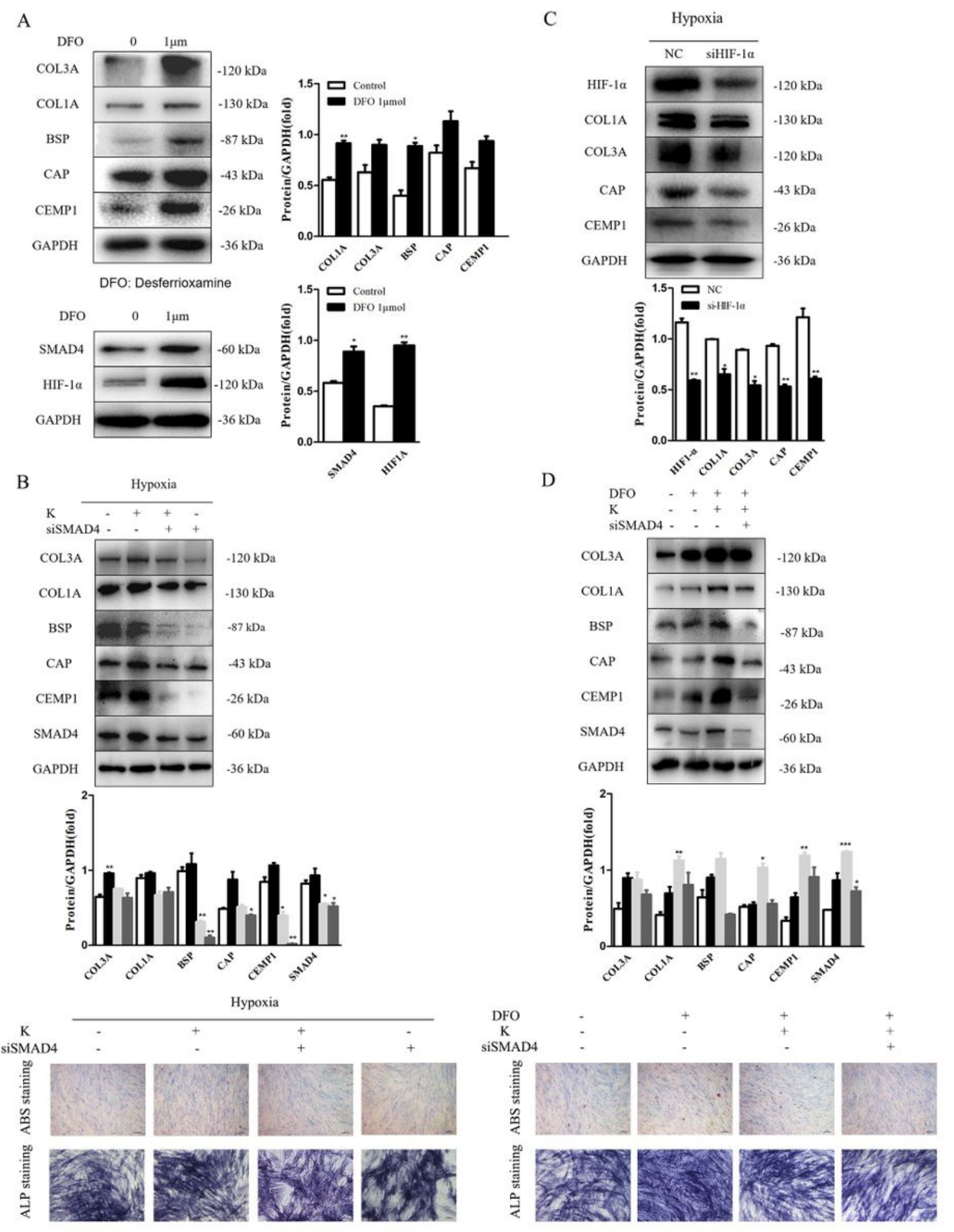

\section{Figure 4}

SMAD4 might be regulated by HIF-1 $a$, thus affecting the cementogenic differentiation of PDLSCs. (A) Western blot showed that the expression levels of COL1A, COL3A, BSP, CAP and CEMP1 were increased after deferoxamine (DFO) stimulation, while the expressions of HIF-1a and SMAD4 were increased. (B) The expressions of COL1A, COL3A, CAP, CEMP1 and SMAD4 increased after KGN stimulation, in the KGN+siSMAD4 group and the siSMAD4 group, the expressions of the proteins were decreased. ALP 
staining and ABS staining results showed that ALP activity was increased in the KGN group, and the number of positive ABS nodules was increased. In the KGN+siSMAD4 group, ALP activity decreased, and ABS staining positive nodules decreased. (C) The expression levels of HIF-1a, COL1A, COL3A, CAP, and CEMP1 were decreased after HIF-1a siRNA treatment. (D) Western blot showed the expression levels of COL1A, COL3A, BSP, CAP, CEMP1 and SMAD4 were increased after treated with DFO alone or DFO combined with KGN, while the corresponding protein expression levels were decreased after treated with siSMAD4, and the staining results were consistent with the protein results. Data are presented as mean \pm $\mathrm{SD} ; * \mathrm{P}<0.05, * \star \mathrm{P}<0.01$ and ${ }^{\star *} \mathrm{P}<0.001$. 
A

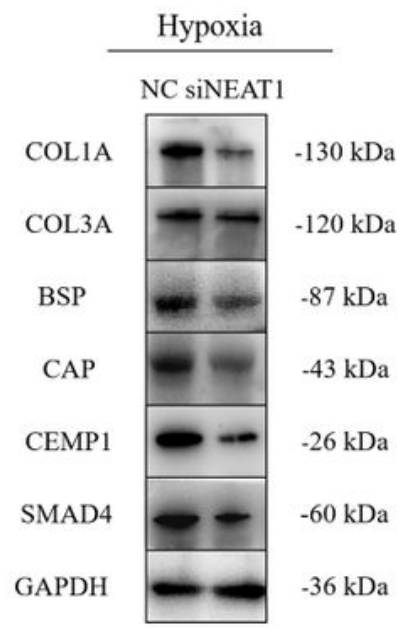

B

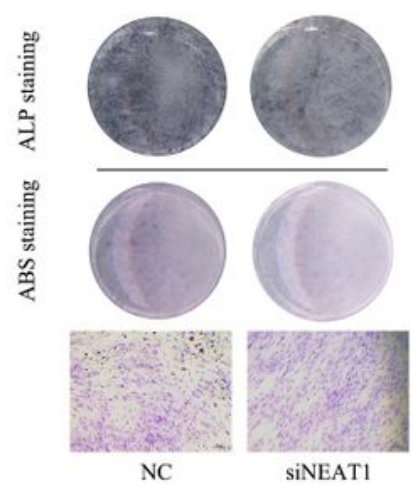

$\mathrm{C}$

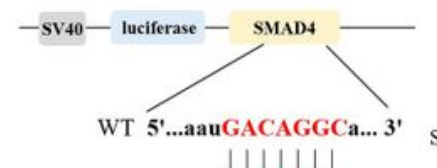
miR-214 3'...cauCUGUCCGu... 5' GAPDH MUT 5 "....auCTGTCCGa... $3^{\prime}$

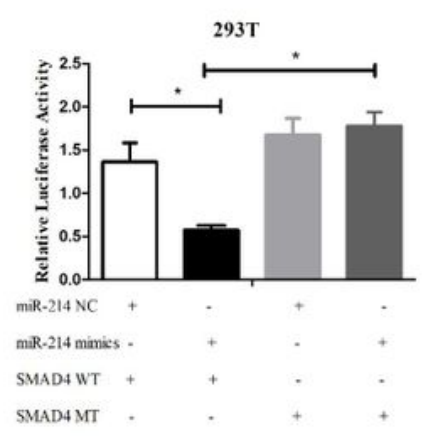

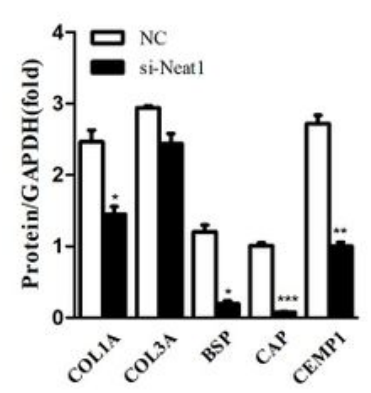

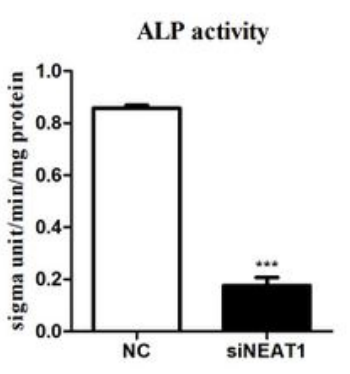

NC mimics iNC inhibitor

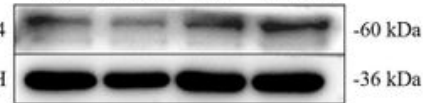
$-36 \mathrm{kDa}$

$\mathrm{F}$

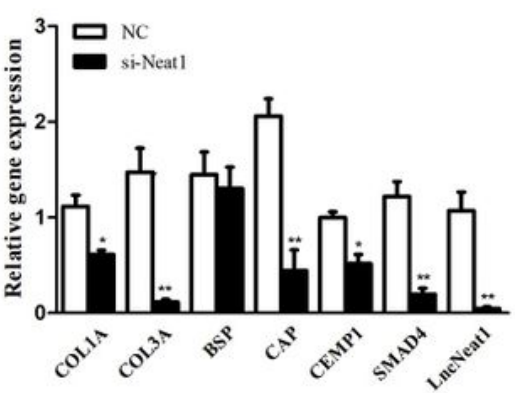

E
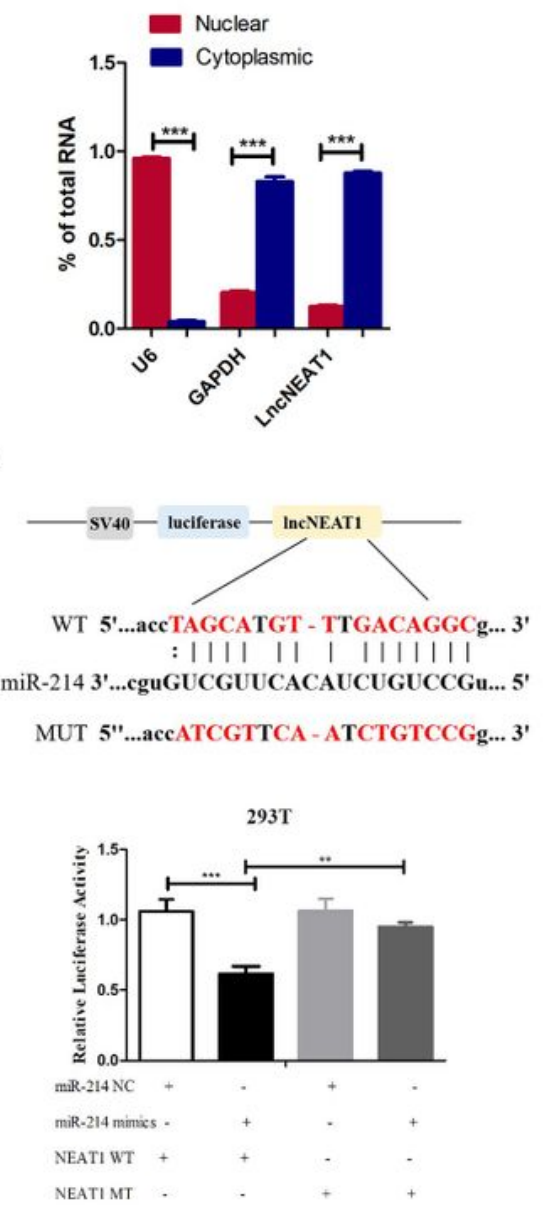

\section{Figure 5}

Lnc NEAT1/miR-214-5p/SMAD4 may constitute a ceRNA mechanism regulating cementogenic differentiation of PDLSCs. (A)(D) Western blot and RT-PCR showed that expressions of COL1A, COL3A, BSP, CAP, CEMP1, and SMAD4 were decreased after NEAT1 siRNA treatment. (B) ALP staining and ABS staining results showed that the ALP activity of siNEAT1 group was decreased and the number of ABS staining positive nodules decreased. (C) Dual-luciferase reporter gene assay was used to verify the 
binding sites between SMAD4 and miR-214-5p, and western blot showed that the expression of SMAD4 decreased when miR-214-5p was overexpressed. (E) Nucleoplasmic separation experiments verified that Lnc NEAT1 was in the cytoplasm. (F) Dual-luciferase reporter gene assay verified the existence of binding site between Lnc NEAT1 and miR-214-5p. Data are presented as mean $\pm S D ;{ }^{*}<<0.05,{ }^{\star *} P<0.01$ and $\star * * P<0.001$.

A

B
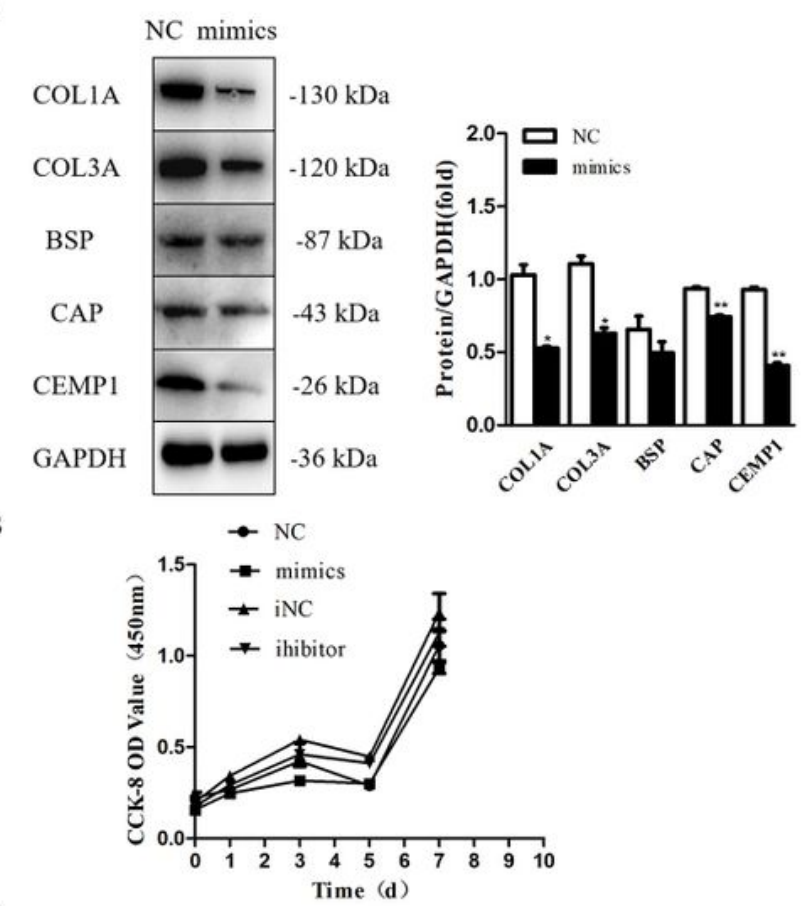

$\mathrm{C}$

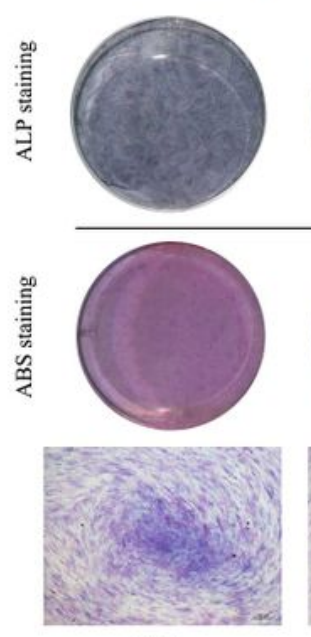

$\mathrm{NC}$
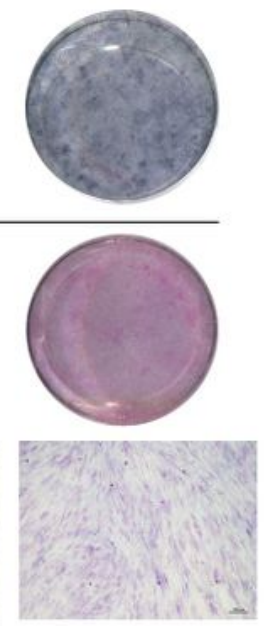

mimics

ALP activity

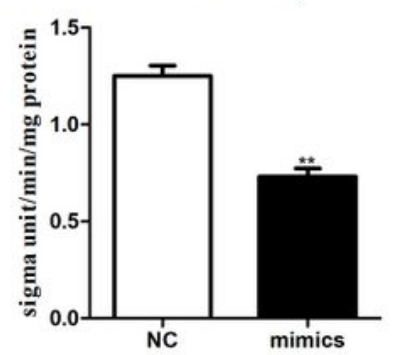

D
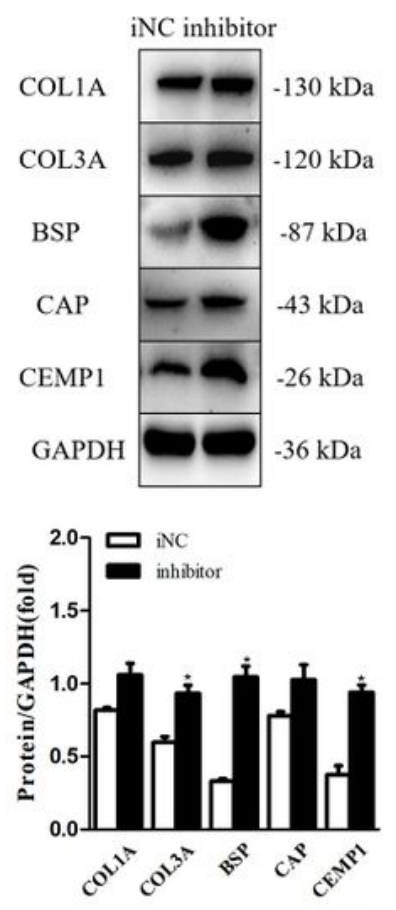

E
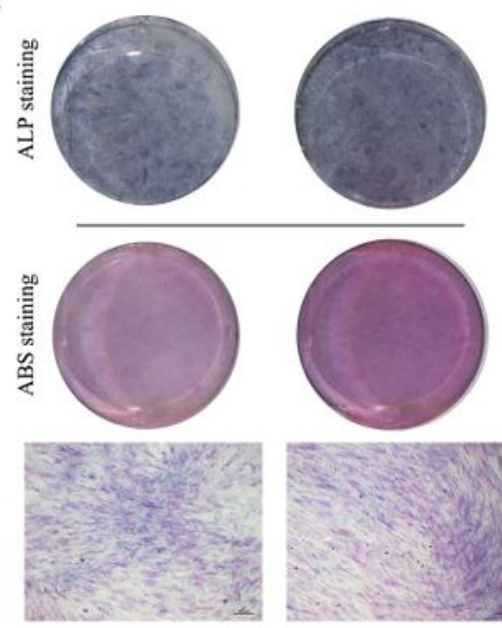

$\mathrm{NC}$
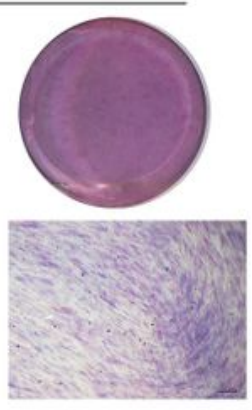

inhibitor

ALP activity

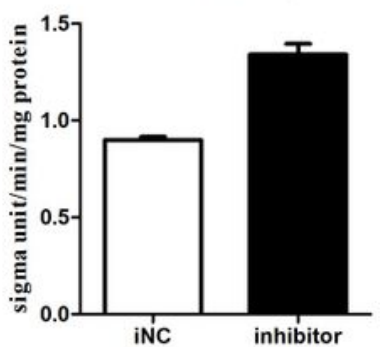

Figure 6 
Lnc NEAT1/miR-214-5p/SMAD4 may constitute a ceRNA mechanism regulating cementogenic differentiation of PDLSCs. (A) Western blot showed that the expression levels of COL1A, COL3A, BSP, CAP, and CEMP1 were decreased after treatment of miR-214-5p mimics. (B) CCK-8 detection showed that miR214-5p mimics, inhibitor and corresponding NC had no significant effect on the proliferation of PDLSCs.

(C) ALP staining and ABS staining results showed that ALP activity was decreased in the mimics group, the number of positive $A B S$ staining nodules was decreased. (D) Western blot showed that the protein expressions of COL1A, COL3A, BSP, CAP and CEMP1 were increased after treatment of miR-214-5p inhibitor. (E) ALP staining and ABS staining results showed that the activity of ALP in the inhibitor group was increased, and the number of positive ABS staining nodules was increased. Data are presented as mean $\pm S D ; * P<0.05,{ }^{*} \mathrm{P}<0.01$ and ${ }^{* * *} \mathrm{P}<0.001$. 
A $\begin{array}{lllll}\text { siNC } & + & - & - & - \\ \text { siNEAT1 } & - & + & + & + \\ \text { miR-214NC } & - & - & + & - \\ \text { miR-214Inhibitor } & - & - & - & +\end{array}$

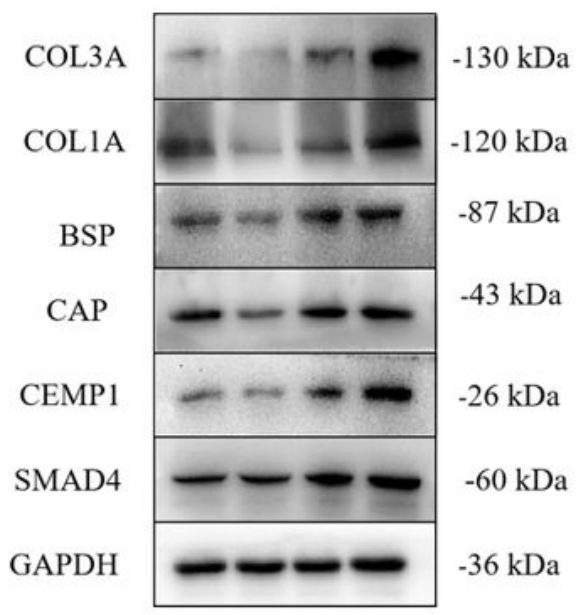

B

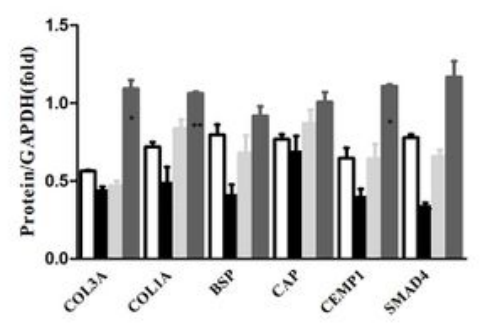

$\mathrm{C}$
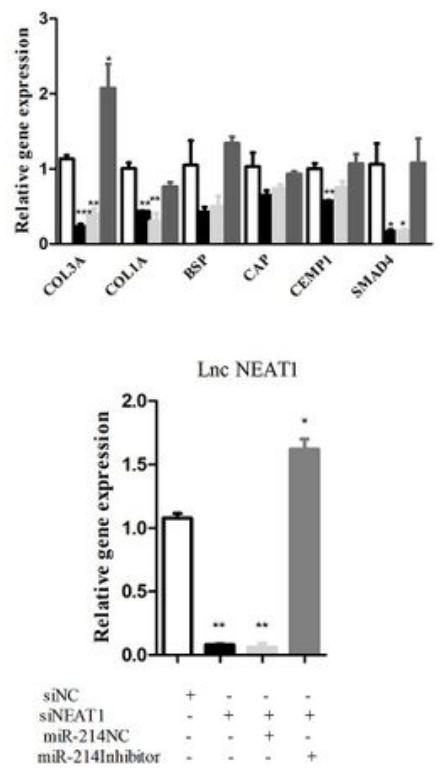
$\begin{array}{lllll}\text { siNC } & + & - & - & - \\ \text { siSMAD4 } & - & + & + & + \\ \text { miR-214NC } & - & - & + & - \\ \text { miR-214Inhibitor } & - & - & - & +\end{array}$

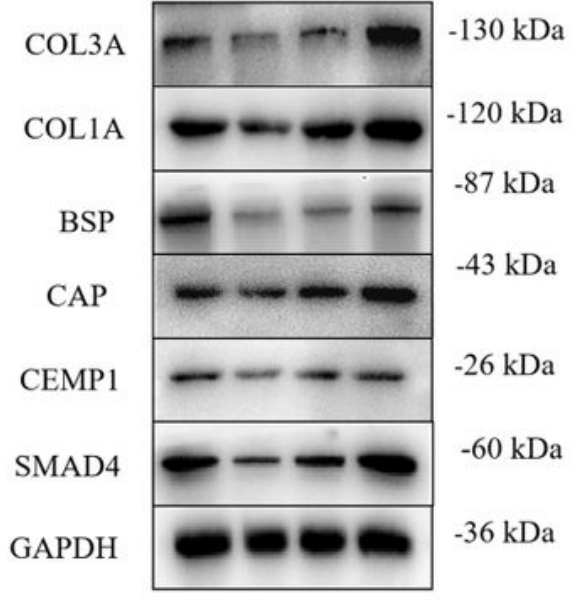

E
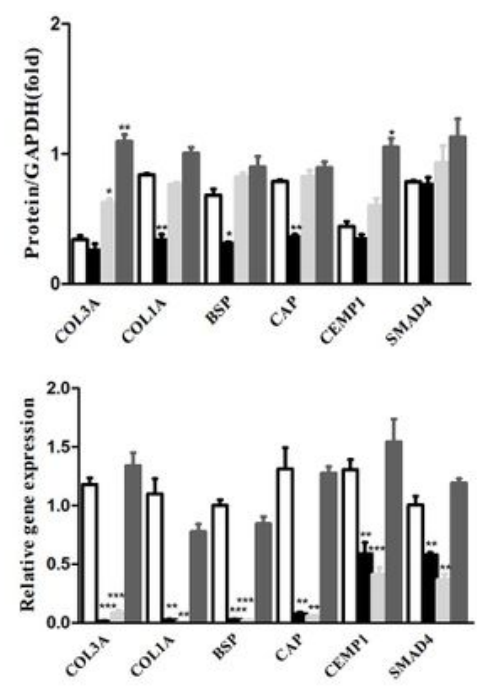

$\mathrm{F}$

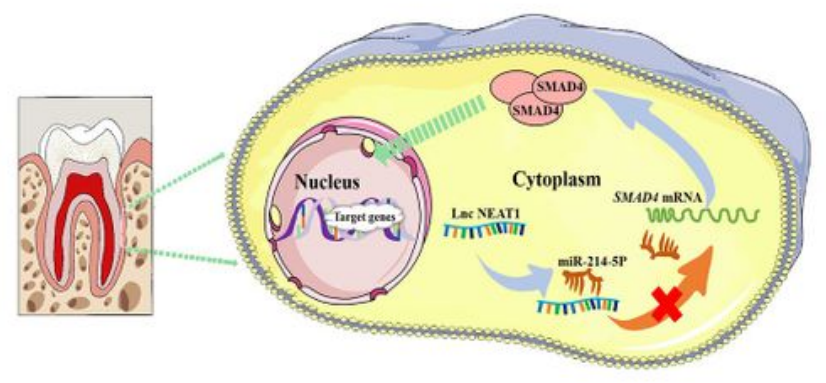

Figure 7

Lnc NEAT1/miR-214-5p/SMAD4 may constitute a ceRNA mechanism regulating cementogenic differentiation of PDLSCs. (A)(B) Western blot and RT-PCR showed miR-214-5p inhibitor could reverse the expression of COL1A, COL3A, BSP, CAP, CEMP1 after siNEAT1 treatment. (C) miR-214-5p inhibitor could reverse the decrease of Lnc NEAT1 gene expression induced by siNEAT1. (D)(E) Western blot and RT-PCR showed miR-214-5p inhibitor could reverse the expression of COL1A, COL3A, BSP, CAP, CEMP1 in PDLSCs 
after siSMAD4 treatment. (F) Experimental mechanism diagram: Lnc NEAT1/miR-214-5p/SMAD4 may constitute the mechanism of regulating PDLSCs cementogenic differentiation. Data are presented as mean $\pm S D ; * P<0.05, * * P<0.01$ and $* \star * P<0.001$.

\section{Supplementary Files}

This is a list of supplementary files associated with this preprint. Click to download.

- FIGURES1.tif

- FIGURES2.tif

- FIGURES3.tif 